\title{
fMRI-Compatible Electronic Controllers
}

\author{
Avrum Hollinger ${ }^{* *}$, Christopher Steele $^{\dagger}$, Virginia Penhune ${ }^{\dagger}$, Robert Zatorre ${ }^{\ddagger *}$, Marcelo Wanderley ${ }^{* *}$ \\ "Input Devices and Music Interaction Laboratory - McGill Schulich School of Music, "Centre for Interdisciplinary \\ Research in Music Media and Technology, ${ }^{\dagger}$ Laboratory of Motor Learning and Neural Plasticity - Concordia \\ University, ${ }^{\ddagger}$ Montreal Neurological Institute \\ Montreal, QC, Canada \\ avrum@music.mcgill.ca
}

\begin{abstract}
This paper presents an electronic piano keyboard and computer mouse designed for use in a magnetic resonance imaging scanner. The interface allows neuroscientists studying motor learning of musical tasks to perform functional scans of a subject's brain while synchronizing the scanner, auditory and visual stimuli, and auditory feedback with the onset, offset, and velocity of the piano keys. The design of the initial prototype and environment-specific issues are described, as well as prior work in the field. Preliminary results are positive and were unable to show the existence of image artifacts caused by the interface. Recommendations to improve the optical assembly are provided in order to increase the robustness of the design.
\end{abstract}

\section{Keywords}

Input device, MRI-compatible, fMRI, motor learning, optical sensing.

\section{INTRODUCTION}

Neuroscientists involved in the study of motor learning of musical tasks (e.g. tapping a rhythm, playing a melody, etc.) use functional magnetic resonance imaging (fMRI) to detect blood flow in the brain, correlating areas of neural activity with cognitive tasks and motor behaviour. An interface that directly captures and logs the physical movement of a subject performing a musical motor task is required to judge the accuracy with which the subject performs the task. In order to synchronize the scanner, auditory and visual stimuli, auditory and visual feedback, and the subjects' interaction with the interface, a system that senses the interaction and communicates that interaction with the other systems is necessary. However, most fMRI studies involving musical motor tasks involve the subject simply moving their fingers and pretending to play without an instrument $[2,6]$ or use a commercially-available electronic instrument which is rendered non-functioning by removing the electronics [9]. In these cases, behavioural data is not directly captured, synchronized, and logged, and the only measures of accuracy come from video observation [4,7]. Other studies measure muscle contraction electromyographically [8] due to the myriad difficulties in

Permission to make digital or hard copies of all or part of this work for personal or classroom use is granted without fee provided that copies are not made or distributed for profit or commercial advantage and that copies bear this notice and the full citation on the first page. To copy otherwise, or republish, to post on servers or to redistribute to lists, requires prior specific permission and/or a fee.

NIME07, June 7-9, 2007, New York, NY

Copyright remains with the author(s). designing a user interface subject to the large magnetic fields set up inside the magnetic resonance imaging scanner environment (MR-environment). Interfaces designed specifically for MRenvironments do not produce (appreciable) artifacts and afford the accurate and synchronized capture of behaviour data, while extending the range of tasks one might study using fMRI. fMRIcompatible input devices, such as a piano keyboard capable of sensing key presses, releases, and velocities are needed for studies involving motor learning of musical tasks. This paper first presents the difficulties confronted in designing devices for MRenvironments followed by a description of the fMRI-compatible electronic piano keyboard and two-button mouse prototypes. A discussion of their performance inside the magnetic resonance imaging (MRI) scanner and improvements for future prototypes are presented as well as what this research will mean for the field of electronic musical instrument design. The devices presented herein are initial prototypes for proof of concept and part of a larger project that will see the construction of other fMRIcompatible interfaces.

\section{2. fMRI-COMPATIBILE DESIGN}

An MRI scanner utilizes a static magnetic field and pulsed electromagnetic energy, the amplitudes of which are both extremely intense. Under certain conditions, the nuclei of hydrogen atoms within the human body create a very small, yet measurable, change in the magnetic field. Without delving into a discussion of how an MRI scanner actually generates images, we can begin to understand that certain objects might disrupt the fine balance of electromagnetic energy that enables this technology to function reliably and predictably. Briefly, we will deal here with three main issues that must be acknowledged in the design of an MRI-compliant interface (though by no means is this exhaustive and should not be used as a checklist to evaluate if a device is MRI-safe): a) safety issues, b) image artifacts, and c) interface reliability. a) Safety Issues: The first and foremost issue to keep in mind is always the safety of the subject, and any institution will remind technologists (and cleaning staff) that ferromagnetic materials or objects containing ferromagnetic materials within the MR-environment are strictly prohibited. The powerful static magnetic field is capable of pulling a ferromagnetic object (like an oxygen tank or vacuum cleaner) from across the room and accelerating said projectile into the bore of the magnet, where the head of a subject would normally be resting during scanning. Coils of wire that are subjected to time-varying magnetic fields generate time-varying electric currents. These currents can cause the conductor to heat up and may result in equipment damage or bodily harm. A current-carrying conductor inside a magnetic field creates a Lorentz force causing unrestrained conductors to accelerate. b) Image Artifacts: MR images are susceptible to a 
variety of artifacts, notably subject movement and foreign objects, and (changes in) electric and magnetic fields. We will disregard any motion of the device or the subject for the moment by assuming they are both restrained. Current-carrying conductors and conductors which could carry current (loops of wire and inductors) in the presence of time-varying magnetic fields are liable to distort the magnetic field, especially when they are in close proximity to the bore of the magnet (where the scanner is "focused"), thus causing image artifacts. Therefore electrified devices, wires carrying currents to and from an adjacent room, or simply pieces of metal that form a closed circuit could all be sources of image artifacts. c) Interface Reliability: An interface within the MR-environment, that transmits or receives electronic signals yet does not produce significant image artifacts, may fail due to induced noise caused by the pulsed electromagnetic field of the scanner. This is more likely in the more powerful $3 \mathrm{~T}$ (three Tesla) MRI scanners. That is, devices which worked in the $1.5 \mathrm{~T}$ scanners may not be compatible with newer 3T machines, both in terms of artifacts and electromagnetic interference.

\subsection{Existing MRI-Compatible Devices}

There has been some work done in the field of MRI-compatible input devices, including the study of commercially-available devices modified for MR-environments and the design of custombuilt interfaces. Certain commercially-available computer keyboards have even been tested and performed adequately in $3 \mathrm{~T}$ scanners without any modification [5]. Testing using the buttons of a standard computer mouse gives accurate behavioural data, but studies conducted on $1.5 \mathrm{~T}$ scanners may not be portable to $3 \mathrm{~T}$ systems [3]. Takahashi et al. have developed a novel 6-axis force sensor specifically for fMRI studies [11]. Bangert et al. have conducted studies utilizing a custom-built fMRI-compatible electronic piano keyboard [1], however a detailed description of the device was not included.

\section{PROTOTYPE DESIGN}

A portion of the keyboard from an upright piano was salvaged and voided of several ferromagnetic screws, replacing some of them with brass fixtures. The capstans of the original piano, being more than a half-century old, used ferromagnetic pins driven deep into each key and thus the keys had to be truncated. Otherwise, the keyboard contained only non-ferrous materials: wood, felt, brass guide and balance pins, and lead weights. Due to the removal of the capstans and absence of the piano action, the keys were balanced with additional lead weights. The geometry, orientation, and movement of the paramagnetic and conductive materials were judged at the outset to be safe, cause negligible artifacts, and move unimpeded within the MR-environment.

\subsection{Optical Sensing}

Key presses, velocity, and release are sensed optically using an LED $(\lambda=660 \mathrm{~nm})$, a matching phototransistor, a pair of fiber optic cables, and a reflector for each key of the keyboard. To obviate image artifacts (and other aforementioned issues) due to electric currents, all electronics are located in the room adjacent to the MR-environment (dubbed the cold room) and the plastic optical cables are run through the port in the wall to the keyboard within reach of the subject lying supine in the bore of the MRI. The LED and phototransistor housings fix one end of the fiber optic cables in place such that they are properly coupled to the optoelectronic components independent of any movement of the cable or circuit board. The other end of the fiber optic cable pair is secured to the base of the keyboard using a copper gasket and brass screws. The cable pair is aligned in front of a reflector that is attached to the far (capstan) end of the piano key in its depressed state. The reflector is withdrawn when the key is raised. Light emitted by the LED propagates down one fiber optic cable and is only reflected back down the other cable to the phototransistor when the key is depressed (see Figure 1). The phototransistor is biased to provide an output swing of several volts between ambient lighting and optical saturation. This large voltage swing provides overhead to ensure adequate signal-tonoise ratio even with shifts in ambient lighting, obviating the need for compensatory circuitry. The continuous nature of the analog optical sensor enables the measurement of key velocity with a single fiber optic cable pair for each key. As the key is depressed and the reflector moves into alignment, the amount of light reflected onto the sensor increases and the sensor's output voltage increases. The time it takes for the sensor's output to change between two different voltage thresholds can be measured and represents an average velocity over that duration. In this way, not only can note onset velocity be measured, but offset velocity as well (which is an oft-overlooked gestural parameter and lacking in many MIDI keyboards).

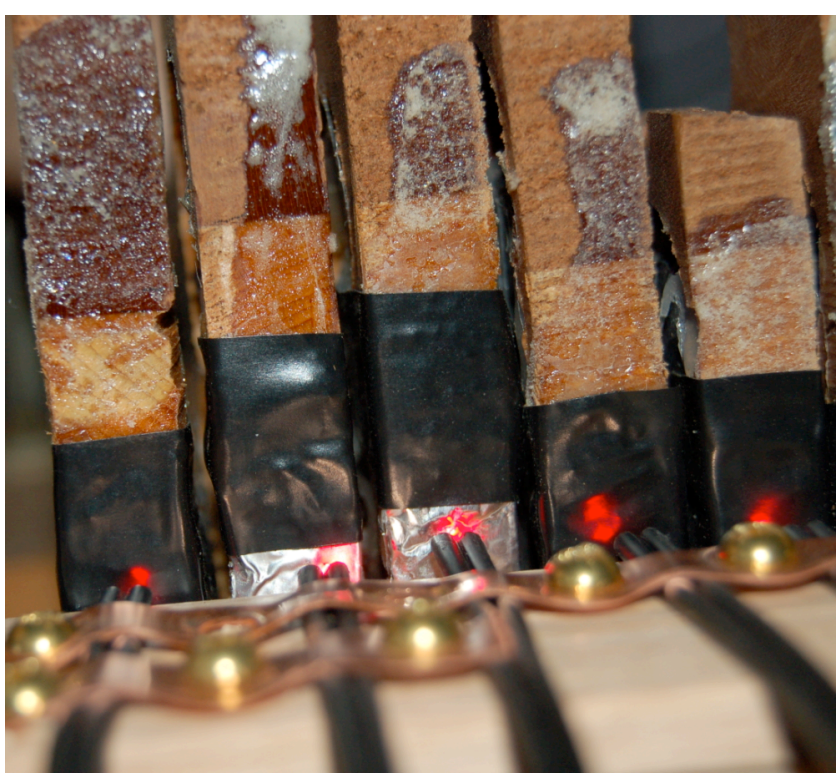

Figure 1. Optical sensing of piano key movement.

\subsection{Hardware Implementation}

A five-volt regulator powers the optoelectronics, comparators, and microcontroller. The circuitry was designed with modularity and key-independent calibration in mind (see Figure 2). The output voltage of each phototransistor is fed to two comparators. The threshold of each comparator is manually tunable with trim pots: one set with a lower threshold voltage representing the start of a key press, and the other set with a higher threshold voltage in accordance with a fully-depressed key. The comparator outputs are wired to interrupt-enabled input pins of the microcontroller, a Cypress Microsystems CY8C27443 programmable system-onchip (PSoC) with an operating frequency of $24 \mathrm{MHz}$. A 32-bit counter is configured onboard the PSoC from which velocity data is obtained. A universal asynchronous receiver/transmitter is configured onboard to receive and send serial data at $31.25 \mathrm{kBd}$. 
The UART output pin is buffered and note on and note off messages are sent conveying note number and velocity using a 5-pin DIN connector as per MIDI specifications [10]. Each PSoC is configured to handle seven piano keys and the chips can be daisy-chained to process additional keys, as the UART input stream is buffered and passed through to the UART output during available cycles.

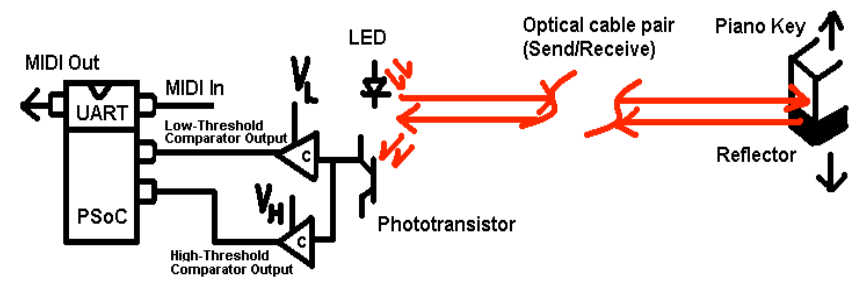

Figure 2. Hardware configuration diagram.

\subsection{Firmware Implementation}

The PSoC firmware was written in $\mathrm{C}$ and assembler, and compiled using Cypress' PSoC designer. The general structure is that of a state machine. Each key can be in one of three states: (1) OFF, (2) partially depressed, (3) fully depressed. In state 1, the key is raised and neither the low nor high optical thresholds have been reached. In state 2 , a sufficient amount of light is reflected to cause a logical high on output of the low-threshold comparator, but insufficient to cause a logical high on the output of the high-threshold comparator. In state 3, the amount of reflected light plateaus causing logical high outputs on both comparators. Understandably, there is no fourth state of high and low logic levels of the low-threshold and high-threshold comparators, respectively. Changes from one state to another occur whenever a comparator's bit is flipped, triggering an input pin interrupt. The interrupt service routine sets a flag indicating a change of state and the input pins are polled to determine which key has been actuated and the status of its comparators. Based on the key's previous state and the new comparator configuration, the keys new state is determined. Assuming a key is in state 1 to begin with, consider what happens when the key then enters state 2: the counter is read and the value is stored as a start time. Next, the key can either be released, in which case the start time is cleared and the key returns to state 1 , or the key can enter state 3 , whereupon the counter is read again to obtain the stop time and a "note on" message is sent corresponding to that key (encoded as the note number) with a velocity proportional to the reciprocal of the difference between the start and stop times. An analogous operation in the opposite direction starting with the key in state 3 produces a "note-off" event, noting that the previous state is always committed to memory whenever the key enters state 2 in order to differentiate between upward and downward-going key transitions. One can see that a key cannot pass directly between states 1 and 3, as well a note event is only reported when the key passes between states 1 and 3, through state 2 . The algorithm assumes that during a piano playing task, the performer will not hold a key in state 2 for extended periods and that she will not press or release the key such that it passes between states 1 and 3 (or back) within one clock cycle (both tasks being near impossible given the physical constraints of the system).

\subsection{Two-Button Computer Mouse}

An fMRI-compatible two-button mouse was also constructed to study rhythmic motor learning tasks (movement and movement tracking were not part of the task). The unmodified computer mouse that had been in use previously with scans done in a $1.5 \mathrm{~T}$ MRI machine was found to be incompatible with the more powerful $3 \mathrm{~T}$ machine due to electromagnetic interference. In addition, the available fMRI-compatible interfaces with buttons did not differentiate between switch depression and release. The interface was comprised of a gutted computer mouse (with the exception of the depopulated circuit board and mechanical switches made of plastic and non-ferromagnetic metal) fixed to a wooden substrate. The interface used the same technology as the piano keyboard described above, though the fiber optic cables were secured by sandwiching them between wooden and acrylic blocks secured with nylon fittings. The only significant difference between the two interfaces was the displacement of the piano keys (on the range of centimetres) versus that of the mouse buttons (on the sub millimetre to millimetre range), and thus the reduced threshold voltage differential.

\section{TESTING AND RESULTS}

The keyboard performed adequately within the MRI scanner during preliminary tests, capturing MIDI voice messages in synchrony with the behavioural stimuli and MR images while causing no apparent image artifacts (see Figure 3). The main issues encountered in testing the device (even before the interface was tested within the scanner) were key-to-key variations in velocity and shifting of optical fiber-reflector alignment in transit. While each key was weighted approximately equally, differences in the felt surrounding the guide pins (due to the keyboard's age) and the asymmetry of the keys resulted in slightly non-uniform movements among the keys. Coupled with the differences in key trajectories and damping, the keys were neither cut to exactly the same lengths nor at a common angle. Thus, it was difficult to mount the reflectors and fiber optic cables to provide consistent light transmission/reflection across all keys. In addition the reflectors used were not meant to be permanent and varied in reflectivity and angle of reflection among keys and along the length of the reflector. Therefore each key had to be calibrated individually in an attempt to approach uniform velocity measurements. Some keys were underdamped and caused nonmonotonic fluctuations in the reflected light during key presses, in the extreme case causing double triggers. Shifting of the fiber optic cables attached to the interface during packaging and transport exacerbated the issues, at times requiring recalibration of the comparator thresholds in situ. Changes in the distance and angle of incidence between the optical cable pair and the reflector have a significant effect on the amount of light transmitted to the photosensor.

\subsection{Recommendations and Solutions}

While proof of concept has been shown with this first prototype, there are many improvements to posit. Firstly, a better quality keyboard with identical key geometries and motion would greatly improve the uniformity of the instrument, from a performance and sensing standpoint. Secondly, the optical system could be greatly improved by treating (systematically cutting and polishing) the ends of the fiber optic cables, adding a proper lens and reflector assembly, and utilizing laser diodes. A lens to focus outgoing and incoming beams and a reflecting Porro prism would allow sharper key transitions providing a more accurate measure of key velocity. Initial testing with a plane reflector and plano-convex lensing has already shown marked improvements in signal-to-noise ratio and 
repeatability. Another option, which would enable key position encoding, is to use of neutral density filters as reflectors with graduated or gradient reflectivites. Precision machined trenches and fasteners to position and fix the optical assemblies are necessary to make the most of the aforementioned additions. Auto-calibrating hardware (electronic pots) and an accompanying PSoC-controlled algorithm would afford additional convenience, accuracy, and uniformity. Onboard sound synthesis is also being considered.

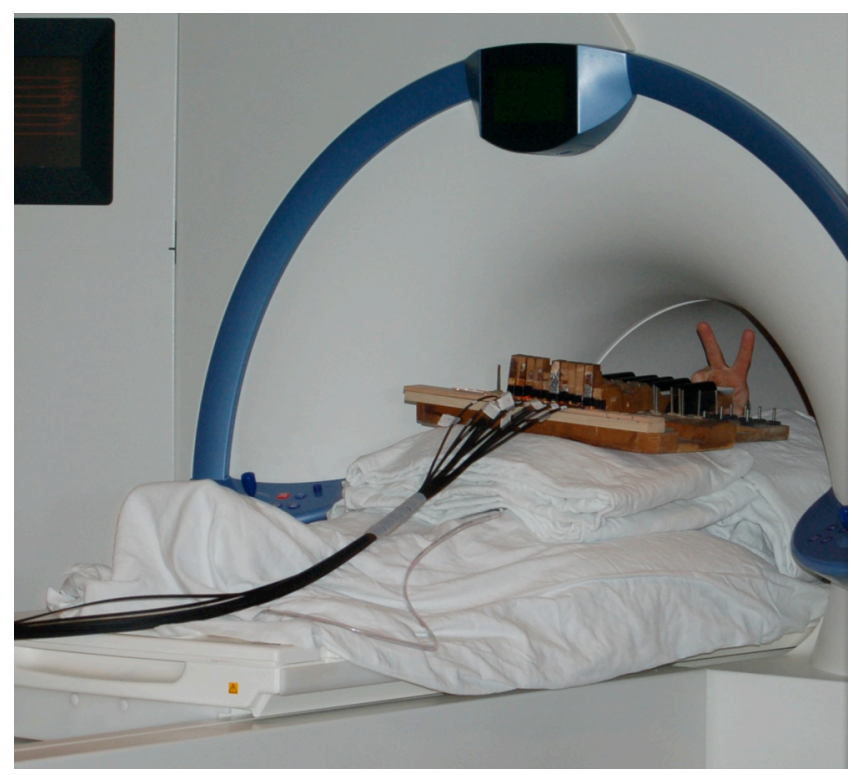

Figure 3. Piano keyboard testing in MRI scanner.

\subsection{MRI-Compatible Mouse Issues}

The fMRI-compatible computer mouse worked adequately in a multi-day study on motor learning of a musical rhythmic task, collecting button onsets and offsets synchronized with visual stimuli and the MRI scanner. No image artifacts due to the interface were measured. The mouse shared the same shortcomings as mentioned with the keyboard above, though with increased sensitivity due to the smaller displacement of the reflector and decreased optical signal-to-noise ratio. In addition, the amount of displacement of the reflector depended on the position of the user's finger upon depressing the underlying switch (which could be felt as a perceivable detent): the switch could be depressed with minimal movement of the reflector in some configurations thus failing to trigger a transition; elsewhere in other configurations simply resting one's finger on the button caused unintended transition triggering. This was rectified prior to the study by applying a tactile finger target to the mouse button so that the subject would push the button in proper position in order to trigger a transition. In this case, a lensed laser diode would provide better spatial resolution and therefore a more robust interface.

\section{LOOKING FORWARD}

Clearly, the interfaces described herein are not the most effective musical instruments to use in a live concert (unless of course you wanted to perform the concert from the comfort of your local MRI scanner - and map brain activation regions to timbral parameters). The interfaces however follow a threefold research paradigm enabling neuroscientists to study the brain during musical performance, developing sensing techniques and systems that might find applications in other interfaces (be they fMRIcompatible or not), and ultimately the synergistic design of novel electronic musical interfaces utilizing the results of neuropsychological studies. If we can better understand the underlying neural processes involved in learning a musical instrument, perhaps we can apply these findings to the development of new electronic musical instruments.

\section{ACKNOWLEDGMENTS}

We would like to thank the Centre for Interdisciplinary Research in Music Media and Technology and our colleagues at the music technology area at McGill for their assistance and feedback. We would also like to thank Allan Hollinger and Alexander Koujelev at the Canadian Space Agency for various discussions on optics and optical sensing. Thank you as well to Michael Ferreira at the Montreal Neurological Institute and Louis Lovsin at Montreal Piano.

\section{REFERENCES}

[1] Bangert, M., et al. Shared networks for auditory and motor processing in professional pianists: Evidence from fMRI conjunction. NeuroImage, 30. (2006), 917-926.

[2] Baumann, S., et al. A Network for Sensory-Motor Integration: What Happens in the Auditory Cortex during Piano Playing without Acoustic Feedback? Ann. N.Y. Acad. Sci., 1060. (2005), 186-188.

[3] Chen, J. L., Zatorre, R. J., and Penhune, V. B. Interactions between auditory and dorsal premotor cortex during synchronization to musical rhythms. NeuroImage, 32 . (2006), 1771-1781.

[4] Itoh, K., et al. Asymmetry of parietal lobe activation during piano performance: a high field functional magnetic resonance imaging study. Neurscience Letters, 309. (2001), 41-44.

[5] James, G. A., He, G., and Liu, Y. A full-size MRIcompatible keyboard response system. NeuroImage, 25. (2005), 328-331.

[6] Jäncke, L., et al. Neural control of playing a reversed piano: empirical evidence for an unusual cortical organization of musical functions. NeuroReport, 17, 4. (2006), 447-451.

[7] Krings, T., et al. Cortical activation patterns during complex motor tasks in piano players and control subjects. A functional magnetic resonance imaging study. Neuroscience Letters, 278. (2000), 189-193.

[8] Lotze, M., et al. The musician's brain: functional imaging of amateurs and professionals during performance and imagery. NeuroImage, 20. (2003), 1817-1829.

[9] Meister, I. G., et al. Playing piano in the mind - an fMRI study on music imagery and performance in pianists. Cognitive Brain Research, 19. (2004), 219-228.

[10] MIDI Manufacturers Association Incorporated. MMA MIDI Specifications. http://www.midi.org (2006).

[11] Takahashi, N., Tada, M., and Ueda, J. An Optical 6-axis Force Sensor for Brain Function Analysis using fMRI. IEEE, 0-7803-8133-5. (2003), 253-258. 